\title{
Copitarsia uncilata Burgos \& Leiva sp. nov. de Cuculliinae para Colombia y México (Lepidoptera, Noctuidae)
}

\author{
Rodrigo Burgos-Pino ${ }^{1}$, Rubén Leiva-Rifo ${ }^{1}$, Andrés O. Angulo² \& Tania S. Olivares ${ }^{3}$
}

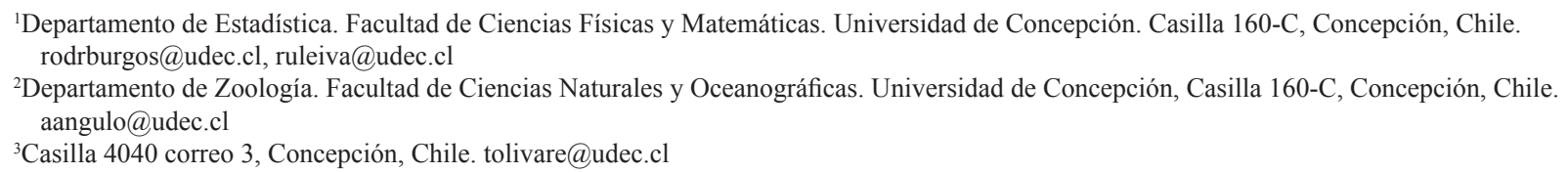

\begin{abstract}
Copitarsia uncilata Burgos \& Leiva sp. nov. of Cuculliinae from Colombia and Mexico (Lepidoptera, Noctuidae). We describe and illustrate the adult and genitalia of male and female Copitarsia uncilata Burgos \& Leiva sp. nov. which is distributed in Colombia and Mexico. The new species is characterized by having a broad spatulated uncus with an apical medium projection, digitus white a wide concave apical emargination and ampulla with recurved apex protruding from the valve. It is externally similar to Copitarsia decolora (Guenée).
\end{abstract}

KEYWORDS. Brassicae oleracea; flower's exportation; Insecta; noctuid moths; taxonomy.

RESUMEN. Copitarsia uncilata Burgos \& Leiva sp. nov. de Cuculliinae para Colombia y México (Lepidoptera, Noctuidae). Se describe e ilustran los adultos y genitalia de macho y hembra de Copitarsia uncilata Burgos \& Leiva sp. nov., que se distribuye en Colombia y México. La nueva especie se caracteriza por poseer un uncus ancho apicalmente espatulado con una diminuta proyección dorsal bulbosa, digitus de corte apical ampliamente cóncavo y una ampulla con ápice recurvado que sobresale de la valva, externamente es similar a Copitarsia decolora (Guenée).

PALABRAS-CLAVES. Brassicae oleracea; flores de exportación; Insecta; polilla; taxonomía.

El género Copitarsia fue descrito por Hampson en 1906. Según la actualización taxonómica hecha por Angulo y Olivares (2003), indica que éste está compuesto por 21 especies, que se encuentran distribuidas desde México hasta la Patagonia. Está asociado a cultivos de importancia económica, tales como, espárragos, alcachofas, maíz y flores de exportación, en los cuales provoca daño cuando se encuentra en estado larval (Castillo \& Angulo 1991; Angulo \& Olivares 2003).

El siguiente trabajo describe una nueva especie del género Copitarsia Hampson, con distribución en Colombia y México.

\section{MATERIAL Y METODOS}

El material revisado corresponde a 15 ejemplares provenientes de Colombia: Jardines de los Andes; Cundinamarca, Sopó, Mun. Chía. (N $4^{\circ} 52^{\prime}$ W $74^{\circ} 04$ ); Cundinamarca, Sopó, San Gabriel (N $04^{\circ} 54^{\prime} 36^{\prime \prime}$ W $72^{\circ} 56^{\prime}$ 21 "); Cundinamarca, Facatativá, Los Moyos ( $04^{\circ} 48^{\prime} 56^{\prime}$ " W 74 21'34") y México: Texcoco $\left(19.30^{\circ} \mathrm{N}, 98.53^{\circ} \mathrm{O}\right)$; Montecillo; Chapingo (N $27^{\circ} 49^{\prime} \mathrm{W} 101^{\circ} 14^{\prime}$ ).

\section{RESULTADOS}

\section{Copitarsia uncilata Burgos \& Leiva sp. nov.} (Figs. 1-4)

Diagnosis: cabeza, tégulas y patagias de color castaño claras con escamas negras y blanquizcas. Ala anterior color general castaño claro con tonalidades brillantes y oscuras, banda media y mancha reniforme poco notorias, banda anterior, posterior transverso y subterminal cubiertas por escamas de color negro, banda terminal representada por manchas triangulares de color negro. Posee valvas con un ensanchamiento en su parte media, dígitus corto y sobresale ligeramente del borde dorsal de la valva, saccus de forma puntiaguda hacia el ápice, uncus alargado angostando paralelamente al llegar a un borde que es ancho, ampulla alargada de ápice romo.

Macho (Fig. 1): ojos con cilias, antenas fasciculadas de color castaño con escamas negras en cada segmento, patagias de color castaño claras con escamas negras concoloras con tégulas y la cabeza, palpos de color negro con escamas castaño claras, frente con escamas blanquizcas. Alas anteriores de color general castaño claras con tonalidades brillantes y oscuras, área basal de color castaño claro mezclada con escamas negras al inicio y centro con una aglomeración de escamas negras, banda anterior cubierta con escamas negras mezcladas irregularmente con escamas de tonalidad brillante, mancha orbicular con borde cubierto con escamas negras y centro con escamas blanquizcas mezcladas con algunas de tonalidad brillante, mancha claviforme ausente, banda media poco notoria, con escamas negras, mancha reniforme cubierta con escamas negras en su borde, aunque no se aprecia en plenitud, banda posterior transversa notoriamente dentada cubierta por escamas de color negro, banda subterminal cubierta por escamas negras, con algunas blanquizcas y brillantes entremezcladas, banda terminal representada por 
manchas triangulares de color negro, mancha apical grisácea con escamas castaño oscuro. Del área basal a la banda media la cubre escamas de color castaño claro, negras y blanquizcas, de la banda media a la subterminal el color general es una tonalidad brillante con escamas negras, de la banda subterminal a la terminal el color es grisáceo con escamas negras. Alas posteriores de color blanco en su interior y borde marginal cubierto por escamas de tonalidad brillante al, igual que su venación. Patas protorácicas (ejemplar de México) de color general castaño claro con bandas negras, patas mesotorácicas de color castaño-claras y oscuras entremezcladas, fémur con escamas castañas, negras y blanquizcas, concoloras con la tibia y el tarso, patas protorácicas (ejemplar de Colombia) con escamas castaño-claras y oscuras entremezcladas, patas mesotorácicas de color castaño claro con escamas negras y blanquizcas mezcladas irregularmente, espolones de color castaño claro con una banda negra en el centro, fémur color castaño claro, con escamas negras y blanquizcas concoloras con la tibia y el tarso, tercer par color general blanquizco con escamas negras y castaño claro, fémur de color blanquizco con escamas negras y castaño claro, tibia y tarso cubiertos con escamas negras, castaño claro y blanquizcas mezcladas irregularmente.

En los ejemplares de México la cabeza, tégulas, patagias $\mathrm{y}$ frente tienen un color castaño oscuro con escamas negras y blanquizcas, la banda anterior, posterior transversa y subterminal son poco notorias, aunque poseen la misma coloración que el ejemplar colombiano, de la banda media solo se aprecia un tercio, la mancha reniforme, al igual que el ejemplar de Colombia, es poco notoria. Las alas posteriores tienen la misma coloración, aunque el borde marginal es más oscuro.

Genitalia del macho (Figs. 2-3): Posee valvas alargadas, 1.2 veces más largo que su ancho medial, con un ensanchamiento en su parte media; dígitus es 3.5 más pequeño que el largo de la ampulla, es corto y sobresale ligeramente del borde dorsal de la valva, con ápice de forma cóncava; yuxta no presenta escotadura, limitada por dos estructuras cortas que asemejan ganchos; saccus es de forma puntiaguda hacia el ápice, cuyo borde posterior es aguzado; uncus alargado de lados subparalelos, cerca del ápice se ensancha y de improvisto y bruscamente se corta, presentando en su centro una pequeña proyección espiniforme; ampulla alargada, de ápice romo, sobresaliendo del borde la valva, cuyo largo es aproximadamente 5-6 veces su ancho medio; presenta un vestigio de clásper que es poco desarrollado, que se encuentra esclerosado; aedeagus posee una vésica que presenta una leve curvatura. Cornuti en base de la vésica, formado por una corona de 14 espinas alargadas similar a otras especies del género Copitarsia, cuyos ápices son puntiagudos, de un largo uniforme; además presenta dos placas de espinas: una de forma triangular muy esclerosada y otra dentada irregularmente con espinas pequeñas situada entre la funda y la vésica.

Hembra: antenas similares a las del macho pero con cilias cortas, aproximadamente la mitad del segmento antenal, alas anteriores con un color general castaño oscuro con escamas negras y de tonalidad brillante, banda posterior transverso y terminal similares a las del macho, mancha orbicular redonda delimitada por escamas negras, el resto de las bandas son poco notorias.

Genitalia hembra (Fig. 4): bursa copulatrix bilobulada, corpus bursae globoso, en el dorso interno presenta signa, formado por numerosas microespinas dispuestas en varias filas que forman una franja que envuelve el corpus bursae, cervix bursae piriforme, globoso desde cuyo ápice se origina el ductus seminalis, ductus bursae esclerosado, sterigma subtriangular muy esclerotizado, apófisis posteriores más cortas y dos veces más anchas que las anteriores.

Envergadura alar: machos, $\mathrm{x}=32.64 \mathrm{~mm}$. $(\mathrm{n}=11)$; hembras, $\mathrm{x}=34.2 \mathrm{~mm} .(\mathrm{n}=5)$.

Distribución geográfica: Colombia: Jardines de los Andes; Cundinamarca, Sopó, Mun. Chía. (N $4^{\circ} 52^{\prime} \mathrm{W} 74^{\circ}$ 04); Cundinamarca, Sopó, San Gabriel (N 04 $54^{\prime} 36^{\prime}$ ' W $72^{\circ}$

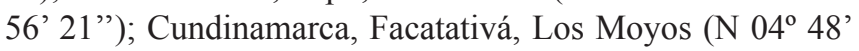
$\left.56^{\prime \prime} \mathrm{W} 74^{\circ} 21^{\prime} 34^{\prime \prime}\right)$; México: Texcoco (19³0' N, 98 $53^{\circ}$ O); Montecillo; Chapingo (N 27 49' W 101 $14^{\circ}$ ').

Etimología: unci=uña, lata=ancho.

Material examinado: (10 machos, 5 hembras): 1 macho, Holotipo, Colombia, 25-07-2007, C.I. Jardines de los Andes, Diana Navarrete Coll. (UCCC) (Copi.15); 1 hembra, Alotipo, Colombia, 25-07-2007, C.I. Jardines de los Andes, Diana Navarrete Coll. (UCCC); 1 macho Paratipo, Colombia, Cundinamarca, Sopó, Mun. Chía., Vda. Fagua, M.G. consultores, N $4^{\circ} 52^{\prime}$ W $74^{\circ} 042562$ m.s.n.m, cría de huevos de postura individual encontrados en cultivos de Alstromeria sp. Macho (Copi.6); 1 macho Paratipo, Colombia, Cundinamarca, Sopó, San Gabriel, finca Suasuque S.A., N 04 $54^{\prime} 36^{\prime \prime}$ W $72^{\circ} 56^{\prime} 21^{\prime \prime} 2600 \mathrm{~m} ., 26$ oct. 2004, P. Venegas (Copi.32); 1 macho Paratipo, Colombia, Cundinamarca, Facatativá, Los Moyos, Km. 20 autopista BogotáMedellín 200m antes del cruce al Rosal, C.F. La Plazoleta Ltda., finca Luisa María, n $04^{\circ} 48^{\prime} 56^{\prime}$ "W 74 21' 34" 2600m., 19 oct. 2004, R. Vera (Copi.31); 1 macho Paratipo, Colombia, Cundinamarca, Facatativá, Los Moyos, Km. 20 autopista Bogotá-Medellín 200m antes del cruce al Rosal, C.F. La Plazoleta Ltda., finca Luisa María, N $04^{\circ} 48^{\prime} 56^{\prime \prime}$ W 74² 21'34" 2600m., 13 oct. 2004, R. Vera (Copi.33); 1 macho Paratipo, Colombia, Cundinamarca, Facatativá, Los Moyos, Km. 20 autopista Bogotá-Medellín 200m antes del cruce al Rosal, C.F. La Plazoleta Ltda., finca Luisa María, N 04 48' 56" W 74 21'34" 2600m., 17 oct. 2004, C. Jara (Copi.30); 1 macho Paratipo, Texcoco Estado de México, Colegio de Postgraduados, entomología agrícola, 27-08-2002, cursiva, cría en Lab., Roberto Flores P. (Copi11); 1 macho Paratipo, Montecillo, Texcoco, Méx., cultivo de col, J. L. Velazco, 12/06/1998, (Copi.10); 1 Paratipo macho, Chapingo, Méx., cultivo de col, Nestor Bautista M., 15/06/1992 (copi 9); 1 Paratipo macho, Chapingo, Méx., cultivo de col, Nestor Bautista M., 15/06/1992; 2 hembras, Paratipos, Texcoco Estado de México, Colegio de Postgraduados, entomología agrícola, 27-28-2002, Copitarsia incommoda, cría en Lab., Roberto Flores P. (\#03,S/ $\mathrm{N}^{\circ}$ ), 2 hembras, Paratipos, Colombia, 25-07-2007, C.I. Jardines de los Andes, Diana Navarrete Coll.,(\#28, \# 30). Todos los tipos depositados en las colecciones científicas de la Universidad de Concepción (UCCC).

En la clave de Castillo \& Angulo (1991), la especie nueva se ubica de la siguiente manera:

2(1) Digitus de ápice truncado subcuadrangular ................ 8

Digitus terminado en punta 3

8. Uncus con ápice ancho apicalmente (espatulado), que termina abruptamente con una pequeña punta ........... C. uncilata Burgos \& Leiva sp. nov.

Uncus ensanchado en la posición medial y terminado en una punta ............................... C. decolora (Gueneé) 

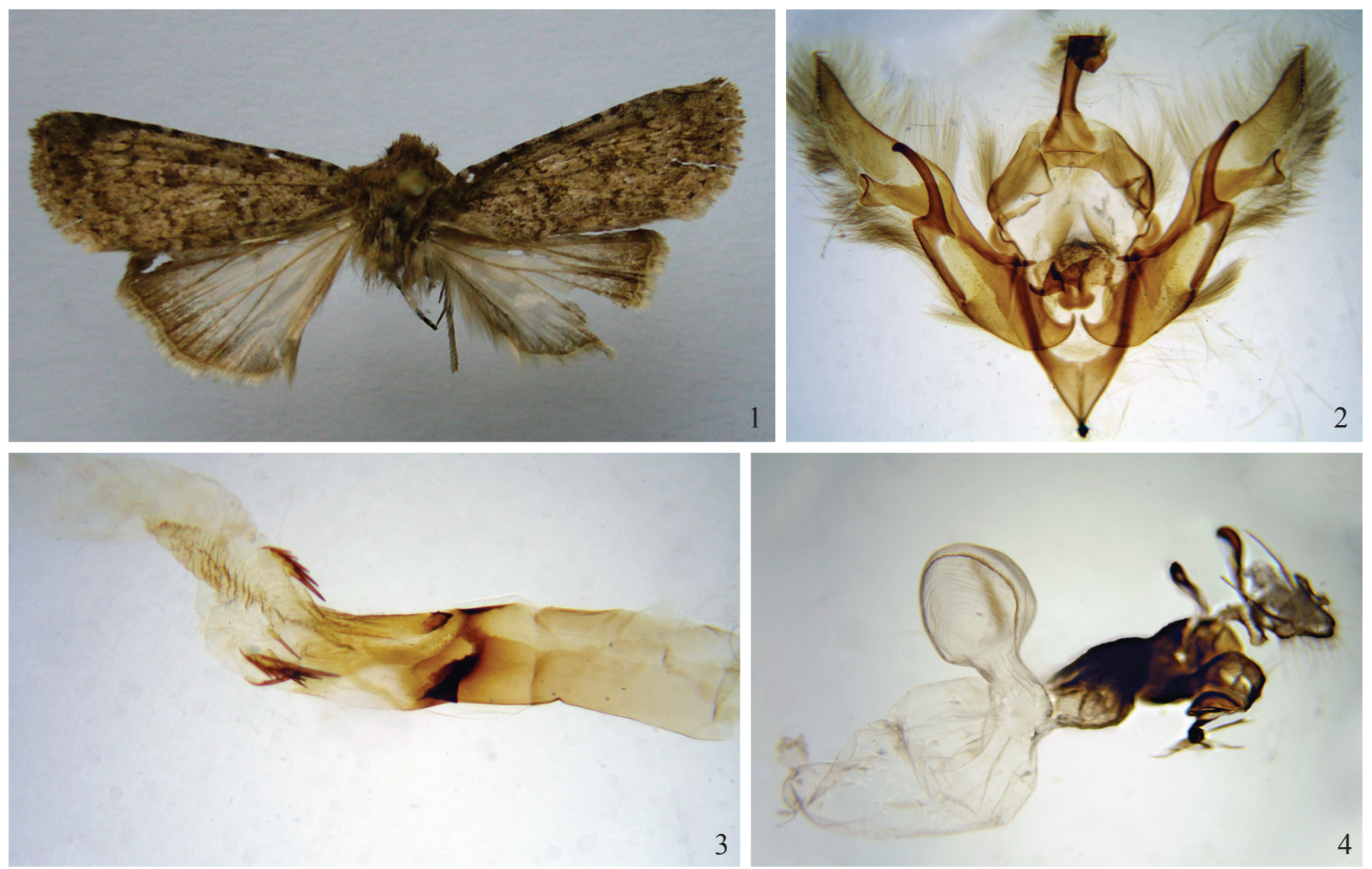

Figs. 1-4. 1, Adulto macho Holotipo; 2, valvas macho; 3, vesica macho; 4, genitalia hembra.

\section{DISCUSION}

Externamente, la maculación alar C. uncilata es similar a C. decolora, C. incommoda y C. corruda por lo que es indispensable la utilización de la preparación de genitalia de los machos para su correcta identificación. En la genitalia masculina son similares en sus valvas, yuxta y aedeagus, pero se diferencian en que C. uncilata posee un uncus ancho apicalmente (espatulado), que termina abruptamente con una pequeña punta, valvas alargadas, corona bien notoria, el dígitus con forma única, y de ápice cóncavo, un saccus puntiagudo hacia el ápice, un tercio del largo de la valva mientras que en $C$. decolora casi 5 veces; ampulla alargada con el ápice algo romo, que sobresale del borde de la valva.

El trabajo de Quimbayo et al. (2010) menciona una variación de la especie Copitarsia decolora asociada a flores, que según este trabajo corresponde a C. uncilata. El material examinado de México para la descripción de esta especie es el mismo que utilizó Flores et al. (2004) en su trabajo donde está asociada a Brassica oleraceae L, al igual que en el trabajo de Suárez-Vargas et al. (2006) la figura 3 de la página 505 corresponde a esta nueva especie.

Existen dos trabajos taxonómicos posteriores a Angulo \& Olivares (2003), que corresponden a Simons \& Pogue, 2004 y Pogue \& Simmons, 2008. En el primer trabajo se aclara las diferencias entre C. decolora y C. incommoda, específicamente en la forma del dígitus, siendo dos especies diferenciables por este carácter; en el otro trabajo se describe una especie nueva, C. corruda Pogue \& Simmons, cuyos hospederos son entre otros Aster, Asparagus, Callistephus, etc menos Alstroemeria; que a nuestro parecer correspondería a una variedad o a lo más una subespecie de C. decolora, debido a que tanto esa forma de digitus es una variación intraespecífica de esta especie. Para el caso de C. uncilata, las diferencias se marcan en el largo de ampulla, forma del dígitus y forma del uncus.

Agradecimientos. Agradecemos al Proyecto de Investigación de la Dirección de Investigación $\mathrm{N}^{\mathrm{o}}$ 207.113.074-1.0 de la Universidad de Concepción por el apoyo logístico y económico a la presente publicación.

\section{REFERENCIAS}

Angulo, A. O. \& T. S. Olivares. 2003. Taxonomic update of the species of Copitarsia Hampson 1906. (Lepidoptera: Noctuidae: Cucullinae). Gayana 67: 33-38.

Castillo, E. E. \& A. O. Angulo. 1991. Contribución al género Copitarsia Hampson, 1906 (Lepidoptera: Glossata: Cucullinae). Gayana Zoología 55: 227-246.

Flores-Pérez, L.; N. Bautista-Martínez; J. Vera-Graziano; J. ValdezCarrasco \& A. O. Angulo. 2004. Ciclo de vida y tasa de supervivencia y reproducción de Copitarsia incommoda Walter (Lepidoptera: Noctuidae) en tres cultivares de Brassica oleracea L. Agrociencia (México) 38 $517-523$. 
Pogue, M. G. \& R. B. Simmons. 2008. A new pest species of Copitarsia (Lepidoptera: Noctuidae) from the Neotropical region feeding on Asparagus and cut flowers. Annals of the Entomological Society of America 101: 743-762.

Quimbayo, N.; F. Serna; T. S. Olivares \& A. O. Angulo. 2010. Nóctuidos (Lepidoptera) en cultivos de flores colombianas. Revista Colombiana de Entomología 36: 38-46.

Simmons, R. B. \& M. G. Pogue. 2004. Redescription of two often-confused noctuid pests, Copitarsia decolora and Copitarsia incommoda (Lepidoptera: Noctuidae: Cuculliinae). Annals of the Entomological Society of America 97: 1159-1164.

Suárez-Vargas, A. D.; N. Bautista-Martínez; J. Valdez-Carrasco; A. O. Angulo; R. Alatorre-Rosas; J. Vera-Graziano; A. Equihua-Martínez \& M. Pinto V. 2006. Fluctuación poblacional de $C$. decolora (Guénee) y su asociación con crucíferas comerciales. Agrociencia (México) 40: 501-509. 